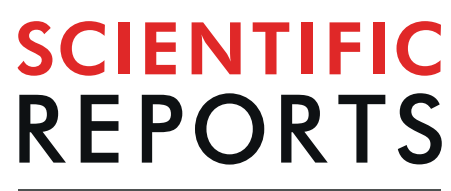

natureresearch

\title{
Effect of tumor necrosis factor inhibition on spinal inflammation and spinal ankylosis in SKG mice
}

\author{
Doo-Ho Lim ${ }^{1,7}$, Eun-Ju Lee ${ }^{2,7}$, Oh Chan Kwon ${ }^{3}$, Seokchan Hong ${ }^{4}$, Chang-Keun Lee ${ }^{4}$, Bin Yoo ${ }^{4}$, \\ Jeehee Youn ${ }^{5}$, Tae-Hwan Kim ${ }^{6}$ \& Yong-Gil Kim $\mathbb{1}^{4 *}$
}

To prevent spinal progression in ankylosing spondylitis, initiating TNF-inhibitor treatment as early as possible is suggested. However, the outcomes are inconsistent in previous clinical studies. Here, we investigated the effect of TNF inhibition alone on spinal progression when used during arthritis development in a murine model. We injected 8-week-old SKG mice with curdlan (curdlan group). We injected adalimumab at 3 and 9 weeks after the first curdlan injection (ADA group). The clinical scores of peripheral arthritis decreased in the ADA group at 3 weeks after first adalimumab injection. Using positron emission tomography-magnetic resonance imaging and histologic examination, spinal inflammation was observed in the curdlan group, and was significantly deceased in the ADA group. However, spinal osteoblast activities by imaging using OsteoSense $680 \mathrm{EX}$ and bone metabolismrelated cytokines such as receptor activator of nuclear factor-kappa B ligand, osteoprotegerin, Dickkopf-1, and sclerostin levels except IL-17A level were not different between the two groups. We conclude that treating TNF inhibitor alone reduced peripheral arthritis score and spinal inflammation in curdlan-injected SKG mice but did not decrease the spinal osteoblast activity, suggesting little effect on spinal ankylosis.

Ankylosing spondylitis (AS) is chronic inflammatory form of arthritis in which the spine and sacroiliac joint are primarily affected. AS is a prototype of spondyloarthritis (SpA) and is characterized by syndesmophytes and the ankylosis of the axial skeleton ${ }^{1}$. Excessive bone formation is an important factor in disease prognosis because it can impair the mobility of the spine. This consequently disturbs daily activity and reduces the quality of life of patients ${ }^{2}$. The exact pathologic process of bone formation in AS is still unclear; however, spinal inflammation may be associated with uncontrolled osteoproliferation that often results in the fusion of the affected joints ${ }^{3}$.

Tumor necrosis factor-alpha (TNF- $\alpha$ ) plays an important role in the inflammatory response in AS. Moreover, TNF- $\alpha$ levels increase in the synovium and serum of AS patients ${ }^{4}$. TNF- $\alpha$ inhibitor treatment clearly improves the functional outcomes, disease activity, and the quality of life of patients who are unresponsive to conventional therapy ${ }^{1}$. However, previous studies have reported inconsistent findings regarding the effect of TNF- $\alpha$ inhibitor treatment on spinal radiographic progression in patients with $\mathrm{AS}^{5-9}$, whereas TNF- $\alpha$ inhibitors have been effective in preventing structural damage as well as reducing disease activity in psoriatic arthritis and rheumatoid arthritis (RA), in which bone erosion dominates bone formation ${ }^{10,11}$.

The roles of TNF- $\alpha$ in bone formation and inflammation in AS are yet to be fully elucidated. Previous in vivo studies demonstrated the uncoupling of bone formation and inflammation in the spine ${ }^{12,13}$ and showed that bone formation was controlled by the bone morphogenetic proteins, transforming growth factors, and Wnt proteins ${ }^{14,15}$. Meanwhile, inflammation is suggested to trigger the initiation of syndesmophytes by an inappropriate repair of inflammatory stress in patients with $\mathrm{AS}^{16}$ as recent clinical studies showed that TNF inhibitors decelerated spinal radiographic progression, especially in patients with early AS without syndesmophytes ${ }^{17-21}$.

${ }^{1}$ Division of Rheumatology, Department of Internal Medicine, Ulsan University Hospital, University of Ulsan College of Medicine, Ulsan, Republic of Korea. ${ }^{2}$ Asan Institute for Life Science, Asan Medical Center, Seoul, Republic of Korea. ${ }^{3}$ Division of Rheumatology, Department of Internal Medicine, Yonsei University College of Medicine, Seoul, Republic of Korea. ${ }^{4}$ Division of Rheumatology, Department of Internal Medicine, Asan Medical Center, University of Ulsan College of Medicine, Seoul, Republic of Korea. ${ }^{5}$ Department of Anatomy and Cell Biology, College of Medicine, Hanyang University, Seoul, Republic of Korea. ${ }^{6}$ Department of Rheumatology, Hanyang University Hospital for Rheumatic Diseases, Seoul, Republic of Korea. ${ }^{7}$ These authors have contributed equally: Doo-Ho Lim and Eun-Ju Lee. *email: bestmd2000@amc.seoul.kr 
a

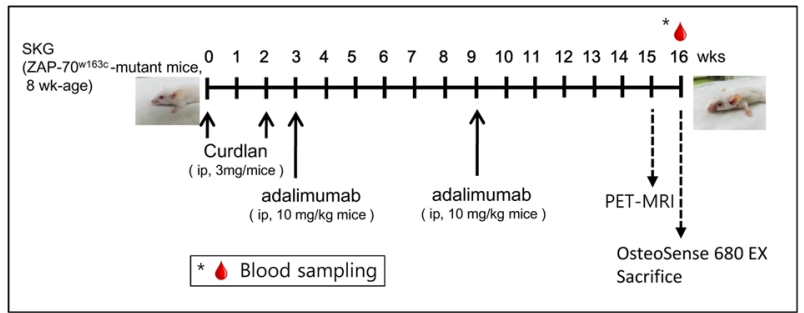

b
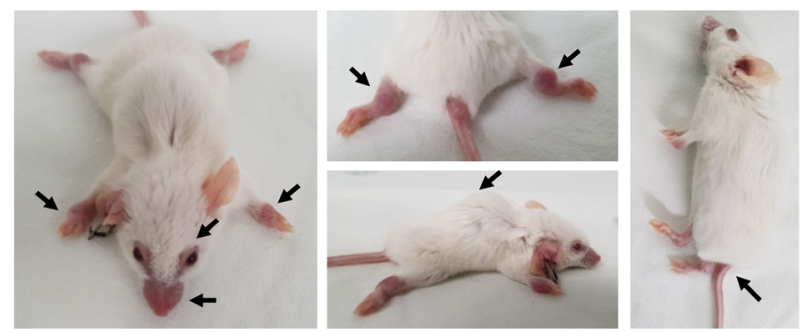

C

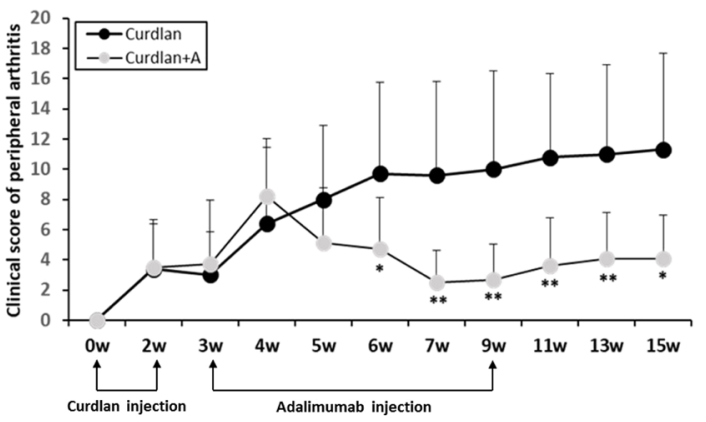

Figure 1. Experimental design and phenotype in curdlan-injected SKG mice. (a) Experimental design. (b) Peripheral arthritis and hair loss around the eye and nose were observed within 2 weeks after injecting curdlan, while tail and spinal deformities were examined 10 weeks after injecting curdlan in SKG mice. (c) Clinical score of peripheral arthritis. Error bars in A indicate mean $\pm \mathrm{SEM} ; * \mathrm{p}<0.05, * * \mathrm{p}<0.01$. Abbreviations: ip, intraperitoneal injection; curdlan $+\mathrm{A}$, curdlan + adalimumab.

However, the effects of TNF inhibitors alone were difficult to verify clearly because standard treatments including nonsteroidal anti-inflammatory drugs were allowed in the clinical studies.

SKG mice develop chronic autoimmune inflammatory arthritis following systemic exposure to ß-glucan ${ }^{22}$. SKG mice harbor a genetic mutation in the SH2 domain of ZAP-70, which is a key signal transduction molecule in T cells ${ }^{23,24}$; as a result, SKG mice have an excess of arthritogenic T cells ${ }^{22}$. This results in chronic arthritis and extra-articular manifestations. Although SKG mice were initially used as a model of RA, Ruutu et al. reported that after systemically injecting curdlan, SKG mice developed the clinical characteristics of SpA, including sacroiliac joint arthritis, vertebral inflammation, enthesitis, peripheral arthritis, uveitis, and bowel inflammation ${ }^{25}$. Therefore, SKG mice can serve as an established animal model for examining the therapeutic effects of TNF inhibition on spinal inflammation.

In this study, we aimed to investigate the effect of a TNF inhibitor administered at the time of peripheral arthritis development on subsequent spinal inflammation and bone formation in an SKG mouse model.

\section{Results}

SKG mice developed spondyloarthritis phenotypes. As shown in Fig. 1b, all SKG mice developed inflammatory arthritis in peripheral joints with soft tissue swelling within 2-3 weeks after the first intraperitoneal injection of 3-mg curdlan, whereas phosphate-buffered saline (PBS)-injected SKG mice did not show any features of inflammation. At 10 weeks after injecting curdlan, redness, swelling, and deformity along the tail and the hunching of the upper body were observed (Fig. 1b).

The effect of adalimumab on peripheral arthritis in curdlan-injected SKG mice was examined. Weekly observations showed that the clinical scores of peripheral arthritis markedly improved in adalimumab-treated mice, beginning at 2 weeks after injecting adalimumab (Fig. 1c). Therapeutic effect on peripheral arthritis in adalimumab-treated mice was sustained for 16 weeks after additionally injecting adalimumab 9 weeks after first injecting curdlan, while the clinical scores of arthritis remained constant in mice without adalimumab treatment. 
$\mathbf{a}$

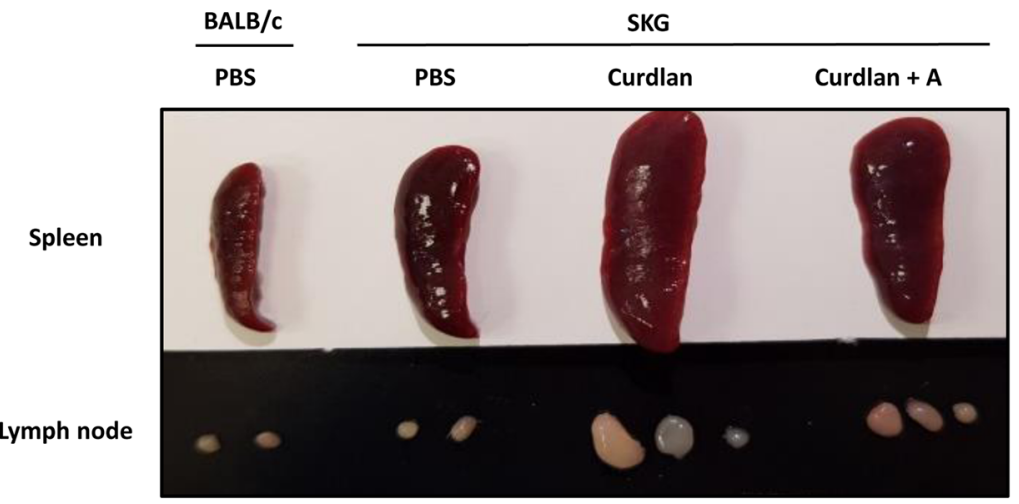

b
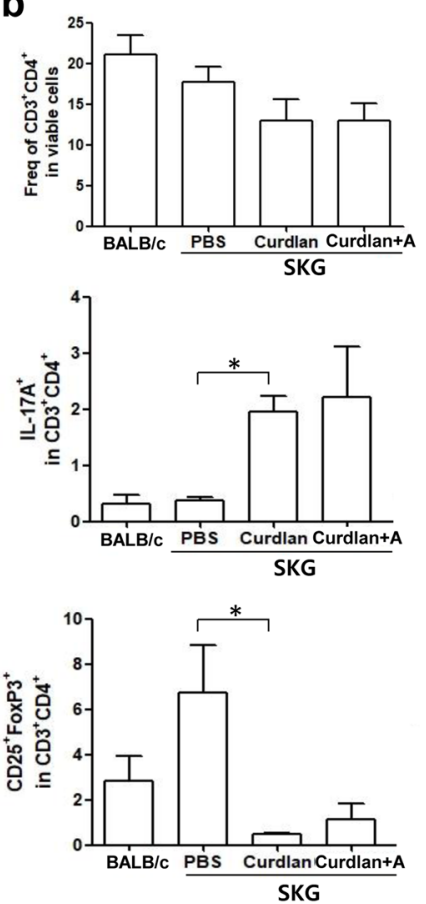
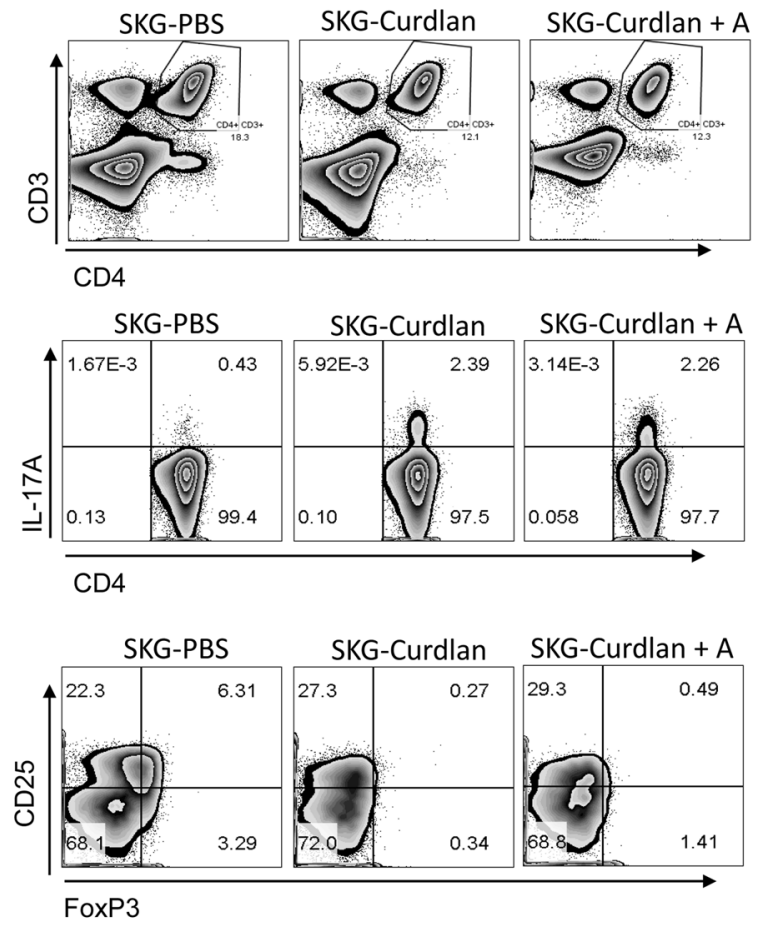

Figure 2. Changes in spleen and T-cell population in SKG mice following curdlan and adalimumab treatment. (a) Gross specimen of spleen and lymph nodes from BALB/c mice, PBS-injected SKG mice, and curdlaninjected SKG mice with or without adalimumab treatment. (b) flow cytometry plots showing the proportion

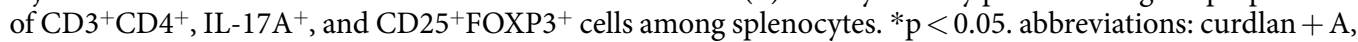
curdlan + adalimumab.

Increased $\mathrm{T}_{\mathrm{H}} 17^{+}$cell population among splenocytes of curdlan-injected SKG mice. To evaluate changes in T-cell population in SKG mice following curdlan and adalimumab treatment, we investigated the characteristics of splenocytes in BALB/c mice, PBS-injected SKG mice, and curdlan-injected SKG mice with or without adalimumab treatment. The size of the spleen and lymph nodes was the largest in curdlan-injected SKG mice without adalimumab treatment (Fig. 2a). Flow cytometry plots showed that there were no significant differences in the frequency of $\mathrm{CD}^{+} \mathrm{CD} 4^{+}$cells $\left(\mathrm{CD} 4^{+} \mathrm{T}\right.$ cells) among the experimental groups (Fig. 2b). There was a significant increase in IL-17A $\mathrm{A}^{+}$cells $\left(\mathrm{T}_{\mathrm{H}} 17\right.$ cells) and a reduction of CD25+ $5^{+}$oxP3 ${ }^{+}$cells $\left(\mathrm{T}_{\text {reg }}\right.$ cells) after curdlan injection. However, the populations of $\mathrm{T}_{\mathrm{H}} 17$ and $\mathrm{T}_{\mathrm{reg}}$ cells were not significantly different between curdlan-injected SKG mice with and without adalimumab treatment (Fig. 2b).

Adalimumab treatment reduced ${ }^{18}$ F-FDG uptake in PET-MRI and inflammatory cells infiltration in spinal tissue. ${ }^{18} \mathrm{~F}-\mathrm{FDG}$ uptake in mice spines using PET-MRI is shown in Fig. 3. Curdlan-injected SKG mice demonstrated higher ${ }^{18}$ F-FDG uptake than PBS-injected SKG mice. However, the quantification of ROIs showed that the SUVR of thoracic spine was significantly reduced in adalimumab-treated mice compared with that in curdlan-injected SKG mice without treatment. These findings were further verified in histologic examination, which revealed the accumulation of inflammatory cells in the para-vertebrae of curdlan-injected SKG mice. The inflammatory lesions were not seen in adalimumab-treated mice. Further, pathogenic lesions in 


\section{$\langle\mathrm{BALB} / \mathrm{C}>$}

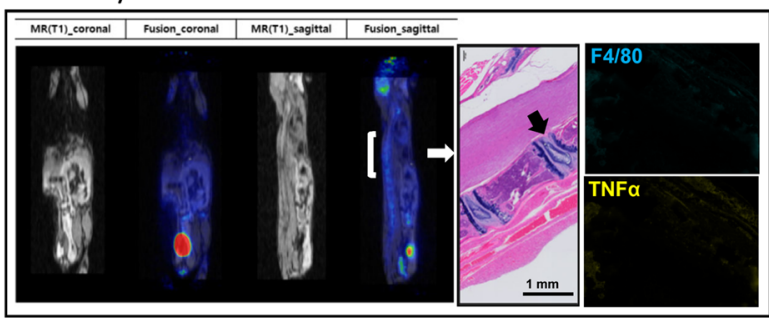

<SKG-Curdlan>

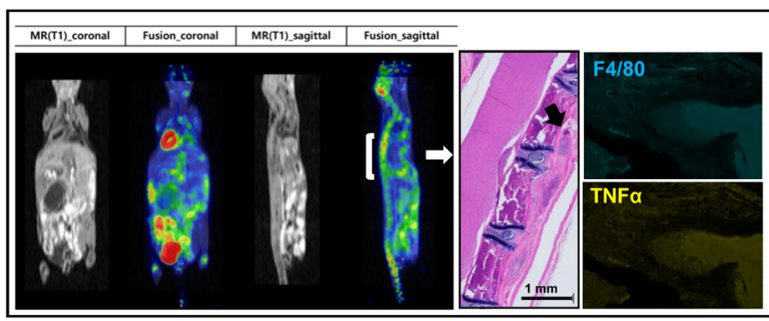

<SKG-PBS>

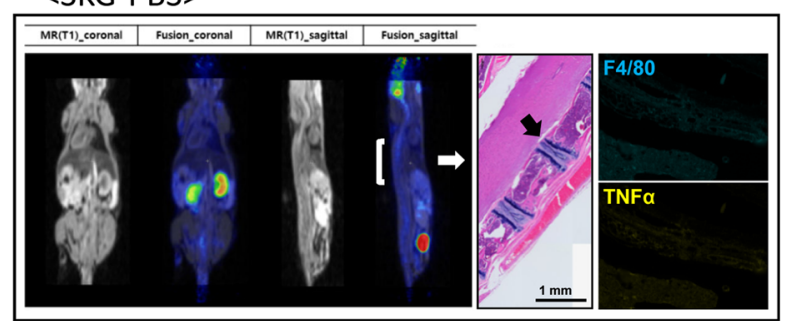

$<$ SKG-Curdlan+A>

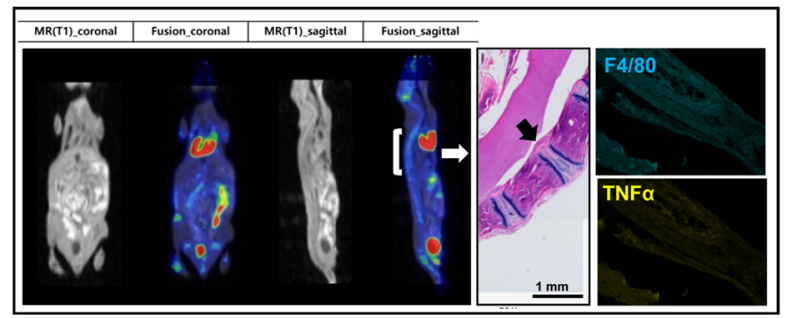

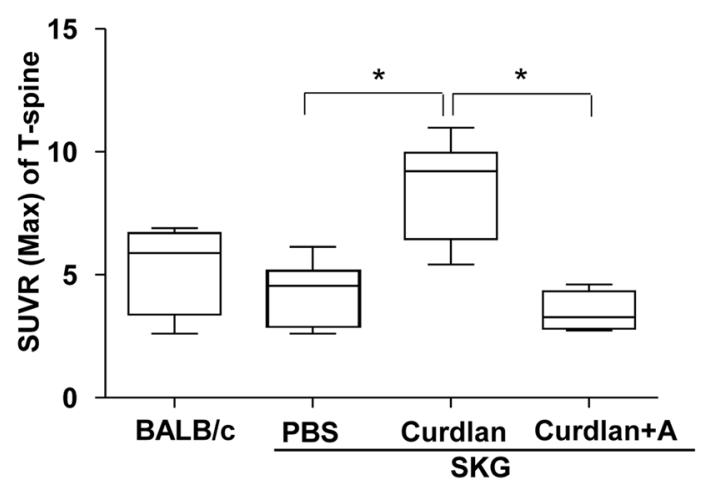

Figure 3. Effect of adalimumab treatment on spinal inflammation. ${ }^{18} \mathrm{~F}-\mathrm{FDG}$ uptake using positron emission tomography (PET), magnetic resonance imaging (MRI), and hematoxylin and eosin staining (white arrow, $\times 200$ ). Immunofluorescence staining (black arrow) images of spinal tissues. ${ }^{*} p<0.05$. Abbreviation: curdlan + A, curdlan + adalimumab.

curdlan-injected SKG mice mainly comprised TNF- $\alpha^{+}$and F4/80 $0^{+}$macrophages. These lesions were decreased in adalimumab-treated mice (Fig. 3).

Adalimumab treatment did not decrease spinal osteoblast activity in curdlan-injected SKG mice. The accumulation of hydroxyapatite that suggested the osteoblast activity was assessed using a fluorescent in vivo bisphosphonate imaging agent (OsteoSense $680 \mathrm{EX}$ ). Figure 4a shows the representative biodistribution of fluorescence signals from the spines of mice. Curdlan-injected SKG mice had higher fluorescence signals than PBS-injected SKG mice, indicating a significant increase in bone formation. However, adalimumab treatment did not attenuate the osteoblast activity, which was further determined using quantitative analysis (Fig. 4a). The serum level of bone metabolism-related cytokines at the time of imaging is shown in Fig. 4b. Serum OPG level was significantly higher in curdlan-injected SKG mice than that in PBS-injected SKG mice, whereas serum RANKL level was not different, suggesting suppressed osteoclastogenic condition in curdlan-injected SKG mice. However, adalimumab treatment in curdlan-injected SKG mice did not restore the serum levels of OPG, RANKL, DKK-1, and sclerostin, supporting the results of imaging. Considering increased $\mathrm{T}_{\mathrm{H}} 17^{+}$cell population among splenocytes of curdlan-injected SKG mice, we additionally measured serum IL-17A levels and demonstrated increased IL-17A level in adalimumab-treated SKG mice compared to the PBS or curdlan-injected SKG mice (Fig. 4b).

\section{Discussion}

Previous clinical studies ${ }^{5-8}$ have shown that TNF inhibition does not prevent spinal radiographic progression in patients with AS, while TNF inhibitors decelerated the progression of structural damages especially in early AS patients without syndesmophyte ${ }^{17-21}$. Additionally, an MRI study revealed that fat infiltration (post-inflammatory changes) may predict bone formation of new syndesmophyte ${ }^{26}$, suggesting the importance of early reduction of spinal inflammation before the potential starting point of new bone formation in AS. However, the protocols of previous clinical studies did not prohibit maintenance of nonsteroidal anti-inflammatory drugs, which are 
a

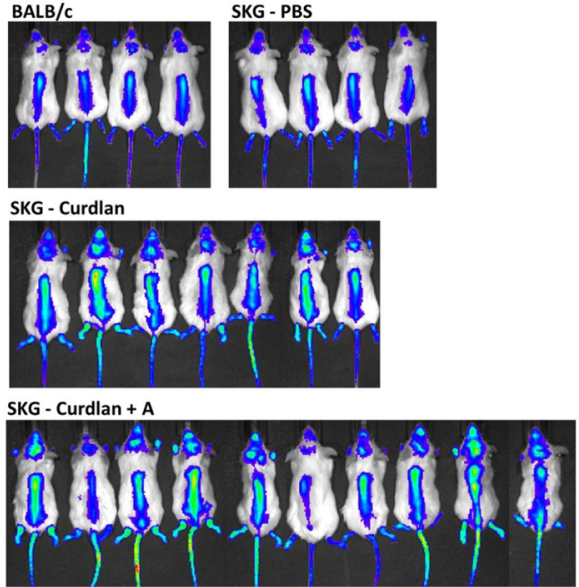

b
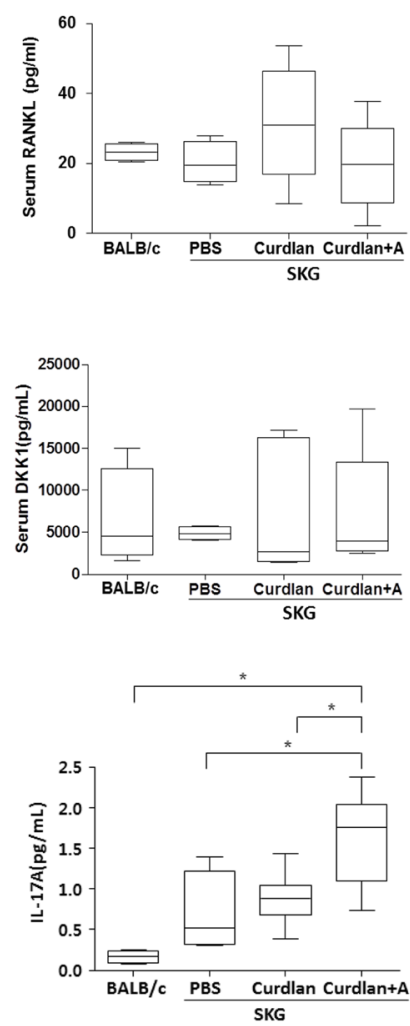
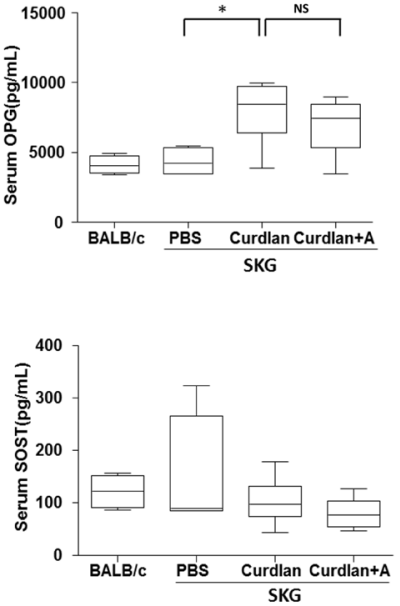

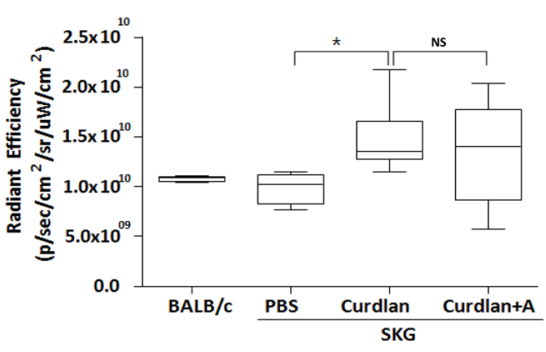

Figure 4. Effect of adalimumab treatment on spinal osteoblast activity. (a) In vivo imaging after injecting OsteoSense $680 \mathrm{EX}$ probe and quantitatively analyzing fluorescence values. (b) The serum levels of bone metabolism-related cytokines and IL-17A in BALB/c mice, PBS-injected SKG mice, and curdlan-injected SKG mice with or without adalimumab treatment. $* \mathrm{p}<0.05$, $* \mathrm{p}<0.01$. Abbreviation: NS, not significant; curdlan + A, curdlan + adalimumab.

difficult to verify only the effects of TNF inhibitors. Therefore, we investigated the effect of TNF inhibition on both spinal inflammation and bone formation as soon as the peripheral arthritis was established.

In this study, we injected adalimumab in the stages of peripheral arthritis (3 weeks after first injecting curdlan), and we observed that TNF inhibition at this point reduced spinal inflammation subsequently in curdlan-injected SKG mice. In addition, inflammatory cell infiltration in the paravertebral tissue was not evident in adalimumab-treated mice. However, TNF inhibition did not decrease spinal osteoblast activity despite a reduction of inflammation in the present study. High osteoblast activity was associated with osteoproliferation and subsequent ankylosis in $\mathrm{AS}^{27}$. Bisphosphonate agent probe (OsteoSense $680 \mathrm{EX}$ ) labeled with near infrared fluorescent bisphosphonate can bind to newly-synthesized hydroxyapatite by osteoblasts ${ }^{28}$; thus, fluorescent intensity indicates osteoblast activity, and subsequent bone proliferation further represents the severity of $\mathrm{AS}^{29}$. Herein, adalimumab treatment did not decrease the osteoblast activity as assessed using fluorescent bisphosphonate imaging, despite of the effect of spinal inflammation.

OPG, which is an extracellular cytokine receptor secreted by mature osteoblasts that promotes bone formation by inhibiting osteoclast activation ${ }^{30}$, was not suppressed by adalimumab treatment in curdlan-injected SKG mice. Furthermore, both serum DKK-1 and sclerostin levels were not influenced by adalimumab treatment, suggesting the existence of other TNF-independent osteoproliferation pathways. In the studies, we found increased serum level of IL-17A in adalimumab-treated SKG mice. IL-17A, a cytokine having osteoclastogenic potency ${ }^{31}$, has been reported to accelerate osteogenesis via enhancing osteoblast differentiation from mesenchymal stem cell populations, and the subsequent activation of the osteoblasts via activation of the JAK2/STAT3 signaling pathway ${ }^{32}$. Walters HM et al. ${ }^{33}$ has also reported increased serum IL-17A in patients with juvenile idiopathic arthritis who were treated by etanercept, a recombinant protein of soluble TNF receptor inhibiting TNF signaling. Therefore, IL-17A pathway induced by adalimumab treatment might be supposed as one of TNF-independent osteoproliferation pathways in addition to the Wnt signaling.

$\mathrm{T}_{\text {reg }} / \mathrm{T}_{\mathrm{H}} 17$ imbalance is associated with the pathogenesis of autoimmune arthritis ${ }^{34}$. It has been reported that $\mathrm{T}_{\mathrm{H}} 17$ cells constitute the key arthritogenic population and are significantly higher in the blood of patients with autoimmune arthritis conditions such as RA, psoriatic arthritis, and $\mathrm{AS}^{35,36}$. The population of $\mathrm{T}_{\text {reg }}$ cells has not been fully evaluated in SpA, but several studies have shown a decrease of $\mathrm{T}_{\text {reg }}$ in the peripheral blood of patients with other autoimmune diseases ${ }^{37-39}$. In addition, previous studies have shown that TNF- $\alpha$ contributed to failure of $\mathrm{T}_{\text {reg }}$ in suppressing the proliferation of effector cells by lowering Foxp 3 mRNA expression, which could be reversed by treatment with TNF- $\alpha$ inhibitors in autoimmune arthritis conditions such as $\mathrm{RA}^{40,41}$. In our study, splenocyte analysis revealed a significant increase in IL-17A ${ }^{+}$cells $\left(\mathrm{T}_{\mathrm{H}} 17\right)$ and a decrease in $\mathrm{CD} 25^{+}$FoxP $3^{+}$cells 
$\left(\mathrm{T}_{\text {reg }}\right.$ ) in curdlan-injected SKG mice compared with those in PBS-injected SKG mice. This finding is consistent with the results of previous studies ${ }^{35-39}$; however, adalimumab treatment did not significantly restore $\mathrm{T}_{\text {reg }} /$ $\mathrm{T}_{\mathrm{H}} 17$ imbalance observed in curdlan-injected SKG mice. A possible explanation for this phenomenon is that the anti-inflammatory effect of TNF inhibitor in this model was mainly mediated by immune cells such as macrophages, mast cells, or neutrophils rather than by $\mathrm{T}$ cells ${ }^{42,43}$.

Our study has several limitations. First, the injection of adalimumab (administered during the stages of peripheral arthritis) may not have been early enough to inhibit spinal bone formation, despite the reduction of spinal inflammation. However, in an actual clinical situation, the time when arthritis is identified could be considered the earliest time to initiate treatment. Second, the mechanism underlying osteoproliferation processes was not clearly identified in this study. As mentioned above, adalimumab treatment did not decrease the osteoblast activity as observed using imaging (OsteoSense $680 \mathrm{EX}$ ); moreover, it did not affect bone metabolism-related cytokine levels. Further studies are warranted to clarify the mechanism of spinal bone formation in SpA. Third, the dose or treatment interval of fully humanized, anti-TNF- $\alpha$ monoclonal antibodies (adalimumab) may not be enough or adequate to demonstrate an inhibitory effect on TNF- $\alpha$ in a murine model. In this study, however, adalimumab treatment improved peripheral arthritis and spinal inflammation. Additionally, previous studies also showed anti-inflammatory effects of adalimumab in several animal models such as female BALB/c $(\mathrm{H}-2 \mathrm{~d} / \mathrm{d})$ rats $^{44}$, collagen-induced mouse arthritis model ${ }^{45}$, Sprague-Dawley rats ${ }^{46}$, and other than human transgenic animal models.

In summary, early treatment using a TNF inhibitor significantly reduced peripheral arthritis and spinal inflammation in curdlan-injected SKG mice; however, it hardly prevented spinal bone formation.

\section{Materials and Methods}

Mice. Female SKG mice with BALB/c background were originally obtained from Dr. S. Sakaguchi (Osaka University, Japan $)^{22}$. Mice were maintained in the specific pathogen-free facility at Asan Institute for Life Sciences (Seoul, Korea). All animal handlings were carried out according to the protocols approved by the Institutional Animal Care and Use Committee of Asan Institute for Life Sciences (2015-14-135).

Experimental schedule. As shown in Fig. 1a, 8-week-old, female, SKG mice were intraperitoneally injected 3 - mg curdlan (Wako, Japan) suspended in 0.2-mL PBS $(\mathrm{n}=20)$ or $0.2-\mathrm{mL}$ PBS alone (control; $\mathrm{n}=5)$ at 0 and 2 weeks. Moreover, 8-week-old, female, BALB/c mice were intraperitoneally injected 0.2 -mL PBS twice (control; $\mathrm{n}=5)$. All mice were monitored for up to 16 weeks. Half of the curdlan-injected SKG mice $(\mathrm{n}=10)$ received adalimumab treatment $(10 \mathrm{mg} / \mathrm{kg}$ of body weight) (fully humanized anti-TNF- $\alpha$ monoclonal antibodies, AbbVie, IL, USA $)^{47}$ at 3 and 9 weeks after the first curdlan injection.

Clinical scoring of peripheral arthritis. Clinical features of peripheral arthritis were monitored weekly and scored for severity on a scale of $0-4(0=$ no swelling, $1=$ mild swelling and redness on the top of the foot, $2=$ severe swelling and redness on the top of the foot, $3=$ severe swelling and redness of the wrist or ankle joints, and $4=$ severe swelling of the wrist or ankle joints and digits $)^{25}$. The scores of affected joints were totaled.

Splenocyte preparation. At 16 weeks after curdlan injection, spleen was filtered through a $100-\mu \mathrm{m}$ nylon filter, and erythrocytes in filtered cells were lysed using red blood cell lysis buffer (BioLegend, CA, USA). The cells were collected by centrifugation. Dead cells were excluded from the analysis with fixable viability dye (eFluor 506; eBioscience, CA, USA). Data of flow cytometry were acquired (FACS Canto II; BD Biosciences, CA, USA) and analyzed (Flow Jo software; Tree Star, OR, USA).

Surface and intracellular staining and flow cytometry. Anti-mouse CD16/32 (BioLegend, clone: 93) was used to block Fc receptors, and the surface markers were stained the following antibodies: FITC-conjugated anti-CD4 (BioLegend, clone: RM4-5), BV421-conjugated anti-CD3 (BioLegend, clone: 145-2C11), and APC/ Cy7-conjugated anti-CD25 (BioLegend, clone: 3C7). After fix and permeabilization, cytokines and transcription factors were stained with the following antibodies: PerCP/Cy5.5-conjugated anti-ROR $\gamma \mathrm{t}$ (BD Biosciences, clone: Q31-378), PE/Cy7-IL-17A (BioLegend, clone: TC11-18H10.1), and Alexa Fluor 647-conjugated anti-FOXP3 (BioLegend, clone: 150D).

Analyzing bone metabolism-related cytokines using luminex multiplex cytokine assay and single cytokine assay. Serum was obtained at 16 weeks after first injecting curdlan. Blood was allowed to clot for a minimum of $1 \mathrm{~h}$ at room temperature (RT) and centrifuged at $16,000 \times \mathrm{g}$ for $15 \mathrm{~min}$ at $4^{\circ} \mathrm{C}$. For measuring bone metabolism-related cytokines including serum osteoprotegerin (OPG), Dickkopf-1 (DKK-1), sclerostin, and the receptor activator of nuclear factor-kappa B ligand (RANKL), the serum concentrations were determined using mouse-bone magnetic bead panel (Milliplex MAP, EMD Millipore Corporation, MA, USA.). Serum diluted with assay buffer (1:1) was mixed with antibody-linked polystyrene beads in 96-well filter-bottom plates and incubated overnight at $4^{\circ} \mathrm{C}$. After washing, the plate was incubated with mouse-bone detection antibody for $1 \mathrm{~h}$ at RT. Without aspiration, streptavidin-phycoerythrin was added and incubated for $30 \mathrm{~min}$ at RT. Subsequently, the plate was additionally washed twice and was resuspended in sheath fluid. All samples were measured in duplicates with standards (7-point dilutions) and a buffer control. Plate was read using the luminex system (Bio-plex 200; Bio-Rad Corp., CA, USA) for quantitative analysis.

For all groups, IL-17A was quantified from serum of mice using Simoa HD-1 Analyzer (Quanterix, MA, USA) at prismCDX Co., Ltd. (Gyeonggi-do, Korea). Limit of detection (LOD) for IL-17A was $0.0110 \mathrm{pg} / \mathrm{mL}$ when compensated for an eight-fold sample dilution. LOD was determined by mean blank signal $+2.5 \mathrm{SD}$. 
Spine histology and Immunofluorescence staining. At 16 weeks after curdlan injection, spines from control and treated mice were fixed in $10 \%$ buffered formalin and embedded in paraffin. Spine tissues were decalcified using ethylenediaminetetraacetic acid. Spine sections $(4 \mu \mathrm{m})$ were cut and stained with hematoxylin and eosin.

Immunofluorescences staining was carried out as previously described ${ }^{48}$. Briefly, using the Opal method (Perkin Elmer, MA, USA), primary antibodies were sequentially applied to each slide. The slides were deparaffinized in xylene and rehydrated in ethanol. Antigen retrieval was performed in citrate buffer ( $\mathrm{pH}$ 6.0) using microwave treatment. Slides were then incubated with primary rabbit antibodies for TNF- $\alpha(1: 500)$ for $1 \mathrm{~h}$ in a humidified chamber at RT, followed by detection using the reagent Opal Polymer HRP Ms + Rb. TNF- $\alpha$ was visualized using Opal 540 (1:100). The slide was again placed in citrate buffer ( $\mathrm{pH} 6.0)$ followed by microwave treatment and then incubated with primary rabbit antibodies for F4/80 (1:500) for $1 \mathrm{~h}$ in a humidified chamber at RT. This was followed by detection using the reagent Opal Polymer HRP Ms + Rb. F4/80 was then visualized using Opal 620 (1:100), and the slide was placed in citrate buffer $(\mathrm{pH}$ 6.0) for microwave treatment. Stained slides were scanned using the multispectral Vectra scanner and quantitative imaging system (Perkin Elmer).

Positron emission tomography-magnetic resonance imaging (PET-MRI). At week 15 after curdlan injection, whole-body sequential PET-MRI of mice was performed (nanoScan PET-MRI; Mediso Ltd, Hungary) (Fig. 1a). The radioligand fluorine 18 -fluorodeoxyglucose $\left({ }^{18} \mathrm{~F}-\mathrm{FDG} ; 0.2 \mathrm{mCi} / \mathrm{kg}\right)$ was injected via the tail vein after a fasting period of at least $12 \mathrm{~h}$. Scanning was initiated at $40 \mathrm{~min}$ after injecting the radioligand. After 20 min of MRI, PET was performed for $10 \mathrm{~min}$. According to the previously described method $^{48}$, contiguous axial slices $(1 \mathrm{~mm})$ were obtained from the whole body. Scanning parameters were as follows: repetition time $=25 \mathrm{~s}$, effective echo time $=3.4 \mathrm{~ms}$, field of view $=64 \mathrm{~mm}$, number of excitations $=1$, frequency $=128$, and phase $=128$. Dynamic data acquisition of PET scans was performed from 60 to $70 \mathrm{~min}$ after injecting ${ }^{18} \mathrm{~F}$-FDG. Acquired PET images were reconstructed using the three-dimensional full detector mode with MRI-based attenuation collection with an energy level of $250-750 \mathrm{keV}$ and $0.5-\mathrm{mm}$ voxel size. Three-dimensional regions of interest (ROIs) were drawn over the joints using a threshold of $70 \%-100 \%$ of the maximum intensity, and the average signal level in the ROIs was measured by a technician. Image counts/pixels were converted to radioactivity concentrations, which were then corrected for the injected radioactivity. Results were expressed as standardized uptake value ratio (SUVR).

Imaging with fluorescent in vivo bisphosphonate agent. At 16 weeks after curdlan injection, whole-body imaging of mice using fluorescent in vivo bisphosphonate agent was performed (OsteoSense $680 \mathrm{EX}$; Mediso Ltd, Hungary). Via the tail vein, mice were injected with the bisphosphonate agent OsteoSense $680 \mathrm{EX}$ (Perkin Elmer) at a dose of $2 \mathrm{nmol} / 100 \mu \mathrm{L}$ PBS per mouse. Back hair was removed on the same day. After $24 \mathrm{~h}$, fluorescent images were obtained using an IVIS spectrum system (Perkin Elmer) with an excitation and emission wavelengths of 675 and 720 , respectively, under inhalation anesthesia (Forane). After acquisition, images were spectrally unmixed (Living Image software; Caliper Life Sciences, MA, USA). ROIs with the same area were placed and measured as the mean radiant efficiency.

Statistical analysis. All statistical analyses were performed using GraphPad Prism 5 (GraphPad Software, La Jolla, CA, USA). For comparisons between two groups, the Mann-Whitney $U$ test was performed. For comparison among multiple groups, one way ANOVA with post-hoc analysis was performed. Results were presented as mean \pm standard error of the mean (SEM). Error bars indicate SEM. P values $<0.05$ were considered statistically significant. $* \mathrm{p}<0.05, * * \mathrm{p}<0.01$.

Received: 3 May 2019; Accepted: 12 November 2019;

Published online: 29 November 2019

\section{References}

1. Braun, J. \& Sieper, J. Ankylosing spondylitis. Lancet (London, England) 369, 1379-1390 (2007).

2. Davis, J. C., van der Heijde, D., Dougados, M. \& Woolley, J. M. Reductions in health-related quality of life in patients with ankylosing spondylitis and improvements with etanercept therapy. Arthritis and rheumatism 53, 494-501 (2005).

3. Sieper, J., Appel, H., Braun, J. \& Rudwaleit, M. Critical appraisal of assessment of structural damage in ankylosing spondylitis: implications for treatment outcomes. Arthritis and rheumatism 58, 649-656 (2008).

4. Francois, R. J., Neure, L., Sieper, J. \& Braun, J. Immunohistological examination of open sacroiliac biopsies of patients with ankylosing spondylitis: detection of tumour necrosis factor alpha in two patients with early disease and transforming growth factor beta in three more advanced cases. Annals of the rheumatic diseases 65, 713-720 (2006).

5. Baraliakos, X. et al. Radiographic progression in patients with ankylosing spondylitis after 2 years of treatment with the tumour necrosis factor alpha antibody infliximab. Annals of the rheumatic diseases 64, 1462-1466 (2005).

6. van der Heijde, D. et al. Radiographic findings following two years of infliximab therapy in patients with ankylosing spondylitis. Arthritis and rheumatism 58, 3063-3070 (2008).

7. van der Heijde, D. et al. Radiographic progression of ankylosing spondylitis after up to two years of treatment with etanercept. Arthritis and rheumatism 58, 1324-1331 (2008).

8. van der Heijde, D. et al. Assessment of radiographic progression in the spines of patients with ankylosing spondylitis treated with adalimumab for up to 2 years. Arthritis research \& therapy 11, R127 (2009).

9. Zong, H. X. et al. Effect of anti-tumor necrosis factor alpha treatment on radiographic progression in patient with ankylosing spondylitis: A systematic review and meta-analysis. Modern rheumatology 29, 503-509 (2019).

10. Keystone, E. C. et al. Radiographic, clinical, and functional outcomes of treatment with adalimumab (a human anti-tumor necrosis factor monoclonal antibody) in patients with active rheumatoid arthritis receiving concomitant methotrexate therapy: a randomized, placebo-controlled, 52-week trial. Arthritis and rheumatism 50, 1400-1411 (2004).

11. Mease, P. J. et al. Etanercept treatment of psoriatic arthritis: safety, efficacy, and effect on disease progression. Arthritis and rheumatism 50, 2264-2272 (2004). 
12. Bardos, T. et al. A longitudinal study on an autoimmune murine model of ankylosing spondylitis. Annals of the rheumatic diseases 64, 981-987 (2005).

13. Lories, R. J. et al. Noggin haploinsufficiency differentially affects tissue responses in destructive and remodeling arthritis. Arthritis and rheumatism 54, 1736-1746 (2006).

14. Lories, R. J., Derese, I. \& Luyten, F. P. Modulation of bone morphogenetic protein signaling inhibits the onset and progression of ankylosing enthesitis. The Journal of clinical investigation 115, 1571-1579 (2005).

15. Diarra, D. et al. Dickkopf-1 is a master regulator of joint remodeling. Nature medicine 13, 156-163 (2007).

16. Schett, G., Landewe, R. \& van der Heijde, D. Tumour necrosis factor blockers and structural remodelling in ankylosing spondylitis: what is reality and what is fiction? Annals of the rheumatic diseases 66, 709-711 (2007).

17. Maas, F. et al. Spinal radiographic progression in patients with ankylosing spondylitis treated with TNF-alpha blocking therapy: a prospective longitudinal observational cohort study. PloS one 10, e0122693 (2015).

18. Molnar, C., Scherer, A. \& Baraliakos, X. TNF blockers inhibit spinal radiographic progression in ankylosing spondylitis by reducing disease activity: results from the Swiss Clinical Quality Management cohort. Annals of the rheumatic diseases 77, 63-69 (2018).

19. Haroon, N. et al. The impact of tumor necrosis factor alpha inhibitors on radiographic progression in ankylosing spondylitis. Arthritis and rheumatism 65, 2645-2654 (2013).

20. Maas, F. et al. Reduction in Spinal Radiographic Progression in Ankylosing Spondylitis Patients Receiving Prolonged Treatment With Tumor Necrosis Factor Inhibitors. Arthritis care \& research 69, 1011-1019 (2017).

21. Park, J. W. et al. Impact of Tumor Necrosis Factor Inhibitor Versus Nonsteroidal Antiinflammatory Drug Treatment on Radiographic Progression in Early Ankylosing Spondylitis: Its Relationship to Inflammation Control During Treatment. Arthritis \&. rheumatology (Hoboken, N.J.) 71, 82-90 (2019).

22. Sakaguchi, N. et al. Altered thymic T-cell selection due to a mutation of the ZAP-70 gene causes autoimmune arthritis in mice. Nature 426, 454-460 (2003).

23. Chan, A. C., Iwashima, M., Turck, C. W. \& Weiss, A. ZAP-70: a 70 kd protein-tyrosine kinase that associates with the TCR zeta chain. Cell 71, 649-662 (1992)

24. Negishi, I. et al. Essential role for ZAP-70 in both positive and negative selection of thymocytes. Nature 376, 435-438 (1995).

25. Ruutu, M. et al. beta-glucan triggers spondylarthritis and Crohn's disease-like ileitis in SKG mice. Arthritis and rheumatism 64, 2211-2222 (2012).

26. Chiowchanwisawakit, P., Lambert, R. G., Conner-Spady, B. \& Maksymowych, W. P. Focal fat lesions at vertebral corners on magnetic resonance imaging predict the development of new syndesmophytes in ankylosing spondylitis. Arthritis and rheumatism 63, 2215-2225 (2011).

27. Ronneberger, M. \& Schett, G. Pathophysiology of spondyloarthritis. Current rheumatology reports 13, 416-420 (2011).

28. Zaheer, A. et al. In vivo near-infrared fluorescence imaging of osteoblastic activity. Nature biotechnology 19, 1148-1154 (2001).

29. Yang, C. et al. Inhibition of Complement Retards Ankylosing Spondylitis Progression. Scientific reports 6, 34643 (2016).

30. Yao, Y. et al. Synergistic enhancement of new bone formation by recombinant human bone morphogenetic protein-2 and osteoprotegerin in trans-sutural distraction osteogenesis: a pilot study in dogs. Journal of oral and maxillofacial surgery: official journal of the American Association of Oral and Maxillofacial Surgeons 69, e446-455 (2011).

31. Zenobia, C. \& Hajishengallis, G. Basic biology and role of interleukin-17 in immunity and inflammation. Periodontology 200069 , $142-159$ (2015).

32. Jo, S. et al. IL-17A induces osteoblast differentiation by activating JAK2/STAT3 in ankylosing spondylitis. Arthritis research \& therapy 20, 115 (2018).

33. Walters, H. M. et al. The impact of disease activity and tumour necrosis factor-alpha inhibitor therapy on cytokine levels in juvenile idiopathic arthritis. Clinical and experimental immunology 184, 308-317 (2016).

34. Nistala, K. \& Wedderburn, L. R. Th17 and regulatory T cells: rebalancing pro- and anti-inflammatory forces in autoimmune arthritis. Rheumatology (Oxford, England) 48, 602-606 (2009).

35. Jandus, C. et al. Increased numbers of circulating polyfunctional Th17 memory cells in patients with seronegative spondylarthritides. Arthritis and rheumatism 58, 2307-2317 (2008).

36. Pene, J. et al. Chronically inflamed human tissues are infiltrated by highly differentiated Th17 lymphocytes. Journal of immunology (Baltimore, Md.: 1950) 180, (7423-7430 (2008).

37. Jiao, Z. et al. Accumulation of FoxP3-expressing CD4+CD25+ T cells with distinct chemokine receptors in synovial fluid of patients with active rheumatoid arthritis. Scandinavian journal of rheumatology 36, 428-433 (2007).

38. Chen, J. et al. Comprehensive evaluation of different T-helper cell subsets differentiation and function in rheumatoid arthritis. Journal of biomedicine \& biotechnology 2012, 535361 (2012).

39. Noack, M. \& Miossec, P. Th17 and regulatory T cell balance in autoimmune and inflammatory diseases. Autoimmunity reviews 13, 668-677 (2014).

40. McGovern, J. L. et al. Th17 cells are restrained by Treg cells via the inhibition of interleukin-6 in patients with rheumatoid arthritis responding to anti-tumor necrosis factor antibody therapy. Arthritis and rheumatism 64, 3129-3138 (2012).

41. Valencia, X. et al. TNF downmodulates the function of human CD4+CD25hi T-regulatory cells. Blood 108, 253-261 (2006).

42. Appel, H. et al. Analysis of IL-17(+) cells in facet joints of patients with spondyloarthritis suggests that the innate immune pathway might be of greater relevance than the Th17-mediated adaptive immune response. Arthritis research \& therapy 13, R95 (2011).

43. Kruithof, E. et al. Synovial histopathology of psoriatic arthritis, both oligo- and polyarticular, resembles spondyloarthropathy more than it does rheumatoid arthritis. Arthritis research \& therapy 7, R569-580 (2005).

44. Catal, F. et al. A human monoclonal anti-TNF alpha antibody (adalimumab) reduces airway inflammation and ameliorates lung histology in a murine model of acute asthma. Allergologia et immunopathologia 43, 14-18 (2015).

45. Palframan, R., Airey, M., Moore, A., Vugler, A. \& Nesbitt, A. Use of biofluorescence imaging to compare the distribution of certolizumab pegol, adalimumab, and infliximab in the inflamed paws of mice with collagen-induced arthritis. Journal of immunological methods 348, 36-41 (2009).

46. Ma, C. H. et al. Protective effects of tumor necrosis factor-alpha blockade by adalimumab on articular cartilage and subchondral bone in a rat model of osteoarthritis. Brazilian journal of medical and biological research = Revista brasileira de pesquisas medicas $e$ biologicas 48, 863-870 (2015).

47. Binder, N. B. et al. Tumor necrosis factor-inhibiting therapy preferentially targets bone destruction but not synovial inflammation in a tumor necrosis factor-driven model of rheumatoid arthritis. Arthritis and rheumatism 65, 608-617 (2013).

48. Kwon, O. C. et al. IL-17A(+)GM-CSF(+) Neutrophils Are the Major Infiltrating Cells in Interstitial Lung Disease in an Autoimmune Arthritis Model. Frontiers in immunology 9, 1544 (2018).

\section{Acknowledgements}

We would like to thank PET and the optical imaging core facility at the ConveRgence mEDIcine research center, Asan Medical Center, for its technical support. This research was supported by a grant from the National Research Foundation of Korea to YG Kim (NRF-2019R1F1A1059736) and the research fund of Rheumatology Research Foundation to DH Lim (RRF-2016-04). 


\section{Author contributions}

Y.G.K., D.H.L. and E.J.L. designed and conducted experiments. O.C.K., S.H. and C.K.L. performed biology molecular analyses. Y.G.K. and B.Y. supported histological analysis. J.Y. and T.H.K. interpreted nuclear imaging data. D.H.L. and E.J.L. wrote the draft of the manuscript. All authors interpreted the data and approved the final version of manuscript.

\section{Competing interests}

The authors declare no competing interests.

\section{Additional information}

Correspondence and requests for materials should be addressed to Y.-G.K.

Reprints and permissions information is available at www.nature.com/reprints.

Publisher's note Springer Nature remains neutral with regard to jurisdictional claims in published maps and institutional affiliations.

(c) (i) Open Access This article is licensed under a Creative Commons Attribution 4.0 International License, which permits use, sharing, adaptation, distribution and reproduction in any medium or format, as long as you give appropriate credit to the original author(s) and the source, provide a link to the Creative Commons license, and indicate if changes were made. The images or other third party material in this article are included in the article's Creative Commons license, unless indicated otherwise in a credit line to the material. If material is not included in the article's Creative Commons license and your intended use is not permitted by statutory regulation or exceeds the permitted use, you will need to obtain permission directly from the copyright holder. To view a copy of this license, visit http://creativecommons.org/licenses/by/4.0/.

(C) The Author(s) 2019 